\section{Papillary Microcarcinoma}

\author{
Shiro Noguchi · Hiroto Yamashita \\ Shinya Uchino $\cdot$ Shin Watanabe
}

Published online: 11 February 2008

(C) The Author(s) 2008

\begin{abstract}
Background Papillary microcarcinoma (PMC) is increasing in incidence because of diagnosis by ultrasound-guided fine-needle aspiration cytology.

Methods Between January 1966 and December 1995, we treated 6019 patients with papillary cancer; among them, 2070 patients with PMC were studied.

Results PMC is essentially very similar to papillary cancer that is $11 \mathrm{~mm}$ or larger and has a very good prognosis. Smaller tumors and younger patients have a better prognosis. Among PMC, larger tumors $(6-10 \mathrm{~mm})$ recur in $14 \%$ at 35 years compared with $3.3 \%$ in patients with smaller tumors. Patients older than 55 years have recurrence in $40 \%$ at 30 years, with a worse prognosis than younger patients who have a recurrence rate of less than $10 \%$. Extracapsular invasion by the primary tumor also has a higher recurrence rate. The majority of recurrences are in the neck. Therefore, annual ultrasound of the neck is effective for recurrence surveillance.

Conclusion Papillary microcarcinoma is similar to larger papillary carcinomas with tumor characteristics and agebased recurrence rate that extends for many years, justifying long surveillance after surgery.
\end{abstract}

\section{Introduction}

Papillary microcarcinoma (PMC), which is defined as papillary carcinoma of the thyroid that is less than $10 \mathrm{~mm}$

S. Noguchi $(\varangle) \cdot$ H. Yamashita $\cdot$ S. Uchino $\cdot$ S. Watanabe Noguchi Thyroid Clinic and Hospital Foundation, Beppu, Japan e-mail: admin@noguchi-med.or.jp in diameter, is diagnosed with increasing frequency recently due to the extensive use of ultrasound-guided fineneedle aspiration cytology. However, there is no consensus in the management of the disease, resulting in a wide spectrum of responses for this condition ranging from observation without treatment [1] to total thyroidectomy plus radioactive iodine treatment $[2,3]$. These extreme differences may partly be the product of the lack of epidemiologic data about this condition. At the Noguchi Thyroid Clinic and Hospital Foundation, we have been gathering follow-up data on thyroid patients since 1922 and currently perform over 1500 thyroid operations annually. Also, according to Fukunaga and Yatani [4], the incidence of occult thyroid carcinoma is significantly higher in Japan $(28.4 \%)$ and in the Hawaiian Japanese population (24.2\%) when compared with the populations of Canada (6\%), Poland (9.1\%), and Columbia (5.6\%). Here we present our experience with PMC, including a long follow-up period, so that it might help others understand of the nature of this condition.

\section{Patients and methods}

Between January 1966 and December 1995, 6019 patients with papillary thyroid cancer were operated on at our institution. Among them, 371 underwent nonradical surgery; because the precise extent of the tumor was not measurable, they were excluded from this study. Those who concomitantly had other types of malignancies and patients whose operation method or other important information was lacking were also excluded. Of the remaining 4840 patients, 2070 (42.8\%) had PMC. Although multiple autopsy studies have stated that the 
prevalence of PMC with respect to gender is equal, the male-to-female ratio in our sample was 1:8.9 [4].

The age at the time of operation ranged from 11 to 83 years old and the distribution conformed well to a symmetric bell curve around the median age of 47 . Hashimoto's disease was concomitantly seen in 188 patients $(9.1 \%)$ and Graves' disease was concomitant in 824 patients $(39.8 \%)$. Single-variable recurrence-free survival rates were calculated using the Kaplan-Meier method and multivariate risk factors were calculated using Cox's regression hazard model.

\section{Results}

Age

The patient's age at initial surgery was one of the significant risk factors influencing recurrence-free survival by single-variable analysis (Fig. 1). However, age was not a risk factor for recurrence of the disease by Cox's proportional hazard model (Table 1). One explanation for this apparent contradiction is that patients who were operated on when they were 56 years old or older would be 81 years old or older after 25 years of follow-up. Therefore, a large number of patients might have died from causes other than PMC. As the population diminishes, the relative weight of a single recurrence is increased, thus contributing to a significant decrease in recurrence-free survival ratio.

\section{Size of primary tumor}

A primary tumor $1-5 \mathrm{~mm}$ in diameter was seen in 1234 patients. The remaining 836 patients had a maximum primary tumor diameter of $6-10 \mathrm{~mm}$. The $1-5-\mathrm{mm}$ tumor group had an improved recurrence-free survival rate 35 years after primary surgery (Fig. 2).

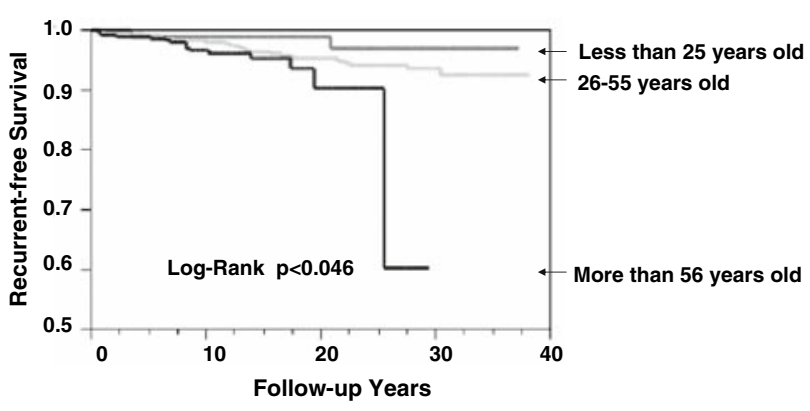

Fig. 1 Recurrence-free survival rates versus patient age between 1966 and 1995. Age was a significant factor in recurrence-free survival by single-variable analysis
Table 1 Cox's proportional hazard model

\begin{tabular}{ll}
\hline Autoimmunity & $p<0.0000$ \\
Gross nodal metastases & $p<0.0043$ \\
Maximum dimension & $p<0.0043$ \\
Adhesion to esophagus & $p<0.0314$ \\
Gender & $p<0.1388$ \\
Age at surgery & $p<0.5094$ \\
\hline
\end{tabular}

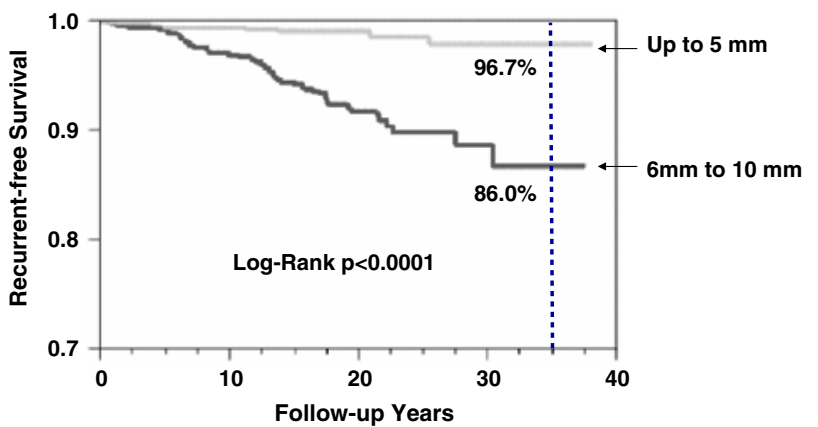

Fig. 2 Recurrence-free survival rates by size of tumor. Tumors up to $5 \mathrm{~mm}$ showed significantly better recurrence-free survival rates 35 years after surgery than tumors between 6 and $10 \mathrm{~mm}$

Autoimmunity

PMC in the presence of autoimmune thyroid diseases showed improved recurrence-free survival 35 years after primary surgery (Fig. 3). The ratio of Hashimoto's thyroiditis to Graves' disease was 1:4.4.

Preoperative diagnosis

When preoperative diagnosis was a benign nodule, the prognosis was slightly better than when preoperative diagnosis was a malignant nodule (Fig. 4). Comparing the preoperative diagnosis regardless of both tumor size and nodal metastasis, patients with Hashimoto's disease or Graves' disease had a better prognosis (Fig. 4).

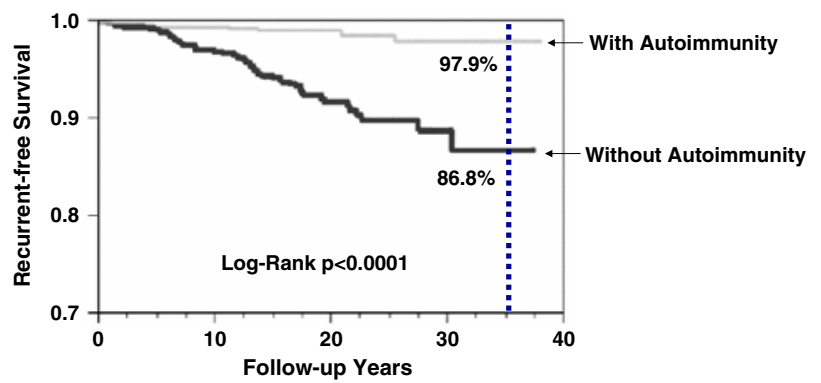

Fig. 3 Recurrence-free survival rates of patients with autoimmunity. Patients with autoimmune thyroid diseases such as Graves' disease and Hashimoto's disease had significantly better prognosis 35 years after surgery than patients without an autoimmune disease 


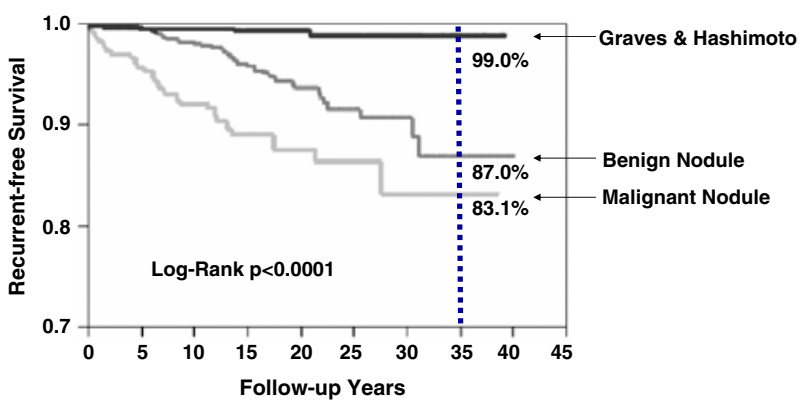

Fig. 4 Recurrence-free survival rates of patients with preoperative diagnosis. Patients preoperatively diagnosed with benign nodules had significantly better prognosis 35 years after surgery than patients preoperatively diagnosed with malignant tumors. Patients whose tumors were not preoperatively identified had the best prognosis

\section{Adhesion}

When the primary tumor had adhered to the recurrent nerve, the recurrence rate of PMC was substantially higher (Fig. 5). Adhesion to the esophagus affected recurrencefree survival more substantially the with recurrence-free survival rate down to $60.5 \%$ (Fig. 6). Adhesion to the recurrent nerve did not significantly affect recurrence by Cox's proportional hazard model, whereas adhesion to the esophagus did.

\section{Nodal metastasis}

There was no significant difference between cases in which gross lymph node metastases were not found and cases in which lymph node metastases were not searched for. However, those who had gross nodal metastases showed a moderately lower recurrence-free survival (Fig. 7). The number of grossly involved lymph nodes was inversely related to recurrence-free survival (Fig. 8), excluding the group in whom the nodal status was not described. This

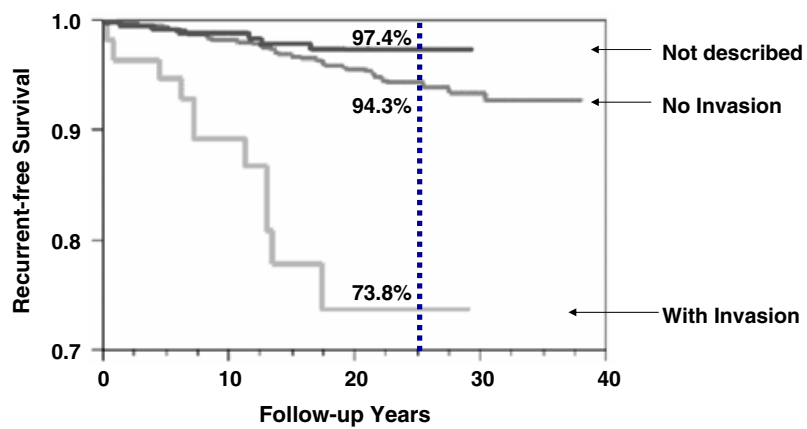

Fig. 5 Recurrence-free survival rates of patients with primary tumor adherent to the recurrent nerve. Patients with documented invasion to the recurrent nerve had significantly worse recurrence-free survival than patients with no documented invasion to the recurrent nerve

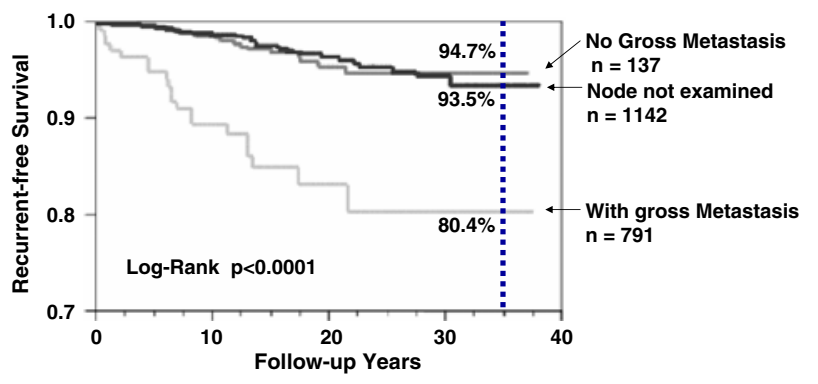

Fig. 6 Recurrence-free survival rates of patients with primary tumor adherent to the esophagus. Patients with documented invasion of the esophagus had significantly worse recurrence-free survival than patients with no documented invasion of the esophagus

group had recurrence-free survival numbers that were similar to the group with no involved lymph nodes (Fig. 7). Of the 246 patients who underwent modified radical neck dissection, metastasis was absent in only 1 . The median number of metastatic nodes was 2 , and the range was 1-43 nodes. Of the 255 patients who underwent central compartment node excision, metastasis was absent in 136 patients $(53.3 \%)$. Fifty-eight patients $(42.6 \%)$ had one metastatic lymph node and 19 patients $(14.0 \%)$ had two metastatic lymph nodes. The remaining patients had up to 14 metastatic nodes $($ mean $=1.1 \pm 2.0$ ).

Other risk factors

This study revealed no significant differences in recurrence-free survival by gender. A benign preoperative diagnosis predicted a slightly better recurrence-free prognosis; however, this difference was small.

Timing of recurrence and sites

The maximum interval between primary surgery and the first recurrence was 31 years (Figs. 9 and 10). There were

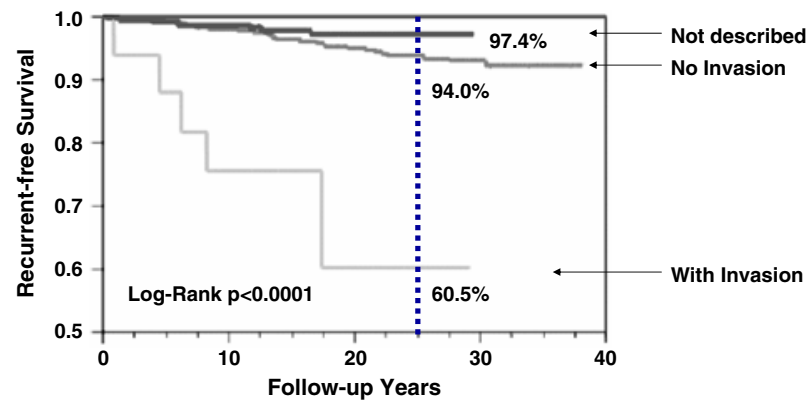

Fig. 7 Patients with documented gross nodal metastases had significantly worse recurrence-free survival than patients with no documented gross nodal metastases 


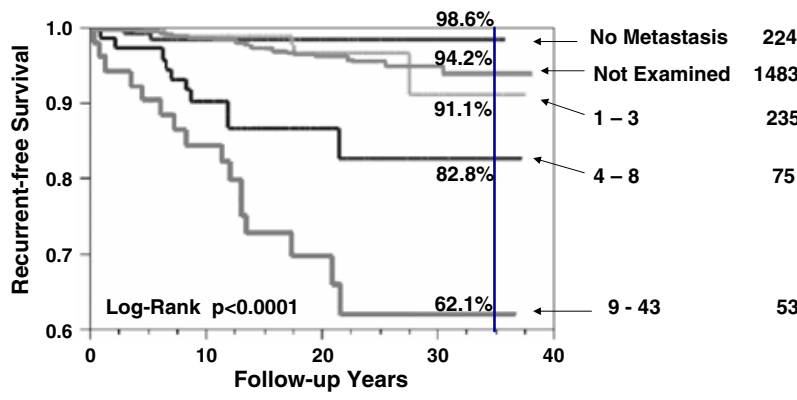

Fig. 8 Numbers of nodal metastases in recurrence-free survival rate. Patients with a large number of nodal metastases had significantly worse recurrence-free survival than patients with fewer nodal metastases

73 patients who experienced recurrence. Median of the interval was 10.29 years and standard deviation was $10.8 \pm 6.51$ years. Second recurrences were seen in 12 patients. One of them had the first recurrence at 0.7 year and the site was contralateral lymph nodes. The second recurrence was in the lungs years after primary surgery and a few weeks after recurrent surgery. This case taught us that careful preoperative work-up is important even with PMC, especially when recurrence occurred after a short interval. The elevated serum thyroglobulin is not always seen with metastasis [5]. Recurrences in remote organs were rare, i.e., five in the lung, five in bone, one in the mediastinum, and one in a miscellaneous site.

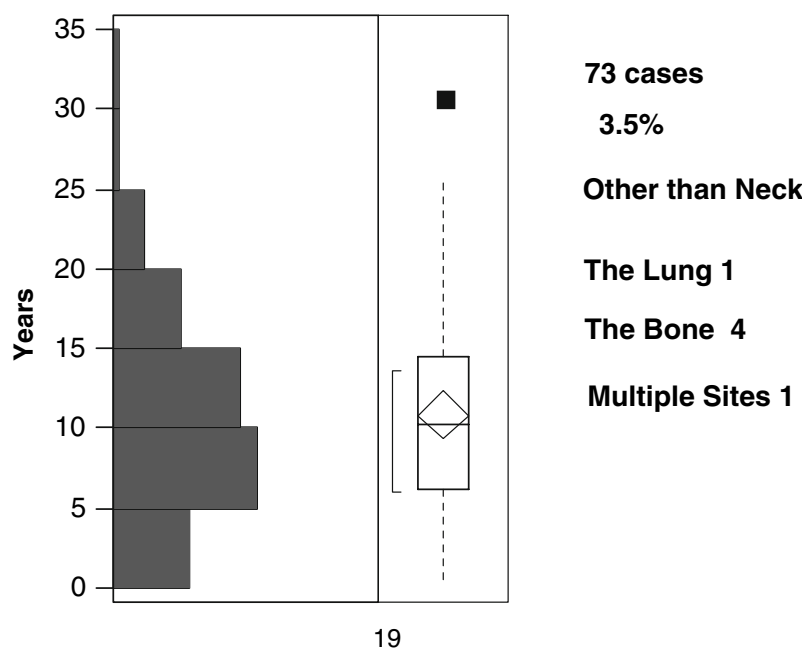

Fig. 9 The interval between primary surgery and the first recurrence. Recurrence was seen in 73 patients $(3.5 \%)$ over a 35 -year period. Among them, 68 patients experienced recurrence in the thyroid bed and/or cervical lymph nodes. One patient had recurrence in the lung, four patients in bone, and one patient in multiple sites. Median interval between primary surgery and first recurrence was 10.29 years. The box represents the median quartile, the diamond represents the average, and the bracket represents the short half

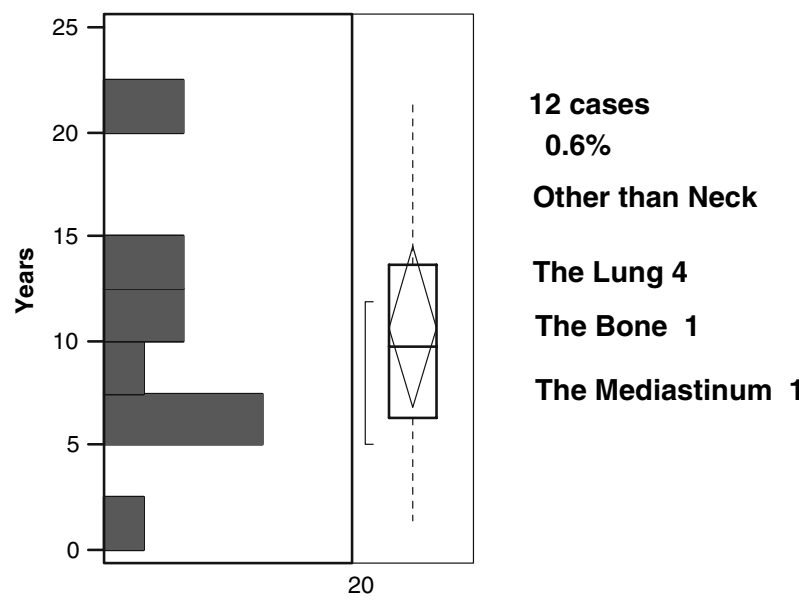

Fig. 10 The interval between primary surgery and the second recurrence. A second recurrence was seen in 12 patients $(0.6 \%)$ over a 35-year period. Four patients had recurrence in the lung, one patient in bone, and one patient in the mediastinum. The box represents the median quartile, the diamond represents the average, and the bracket represents the short half

\section{Discussion}

This article is the largest report of papillary microcarcinoma with long-term follow-up in the literature. The data contained here may help guide diagnostic and therapeutic algorithms for this increasingly common disease.

\section{Difference from past reports}

The differences of this article from our previous report [6] are the proportion of patients with Graves' disease (47.8\% in our previous report and $38.8 \%$ in this study), and the proportion of patients with Hashimoto's thyroiditis $(2.1 \%$ in the previous report and $8.8 \%$ in this report). One reason for the discrepancy may be the use of ultrasonography with increased identification of these small tumors. Another difference between the reports is the patient population included. In this article only patients with pure papillary carcinoma were included, whereas in our previous report a small number of follicular carcinomas, cases of papillary and follicular carcinomas in the same thyroid, and cases of papillary and medullary carcinomas in the same thyroid were included. In this report, the median follow-up period was 15.1 years $($ mean $=16.5$ years $)$ and in our previous report the median was 11 years.

Gender and risk of recurrence

In Japan, PMC is more prevalent in the female population. In this series of patients, the male:female ratio was 1:9. In 
our previous report of small carcinoma of the thyroid, including follicular carcinoma and medullary carcinoma, the ratio was 1:8.4 [6]. This finding is not consistent with the majority of autopsy studies that found no difference in the prevalence of PMC across genders.

\section{Age and risk of recurrence}

Various risk group classifications regard age as one of the most important factors for risk of recurrence [7]. By Cox's proportional hazard model, however, age is not a significant risk factor in this study. (Table 1). Chow et al. [8] also found that age was not a significant factor in predicting disease recurrence or survival for PMC.

\section{Size of PMC and risk of recurrence}

When patients with primary tumors $5 \mathrm{~mm}$ or smaller are compared with patients with primary tumors $6-10 \mathrm{~mm}$, the recurrence-free survival rate at 35 years is significantly better in the group with smaller tumors (Fig. 2). This finding strongly suggests that even among PMC, larger tumors have worse a prognosis compared to smaller ones. In 2004, WHO revised the definition of T1 thyroid carcinoma from $10 \mathrm{~mm}$ to $20 \mathrm{~mm}$. This revision is in conflict with the above finding if recurrence-free survival is considered an important outcome measure.

\section{Autoimmunity}

Vini et al. [9] reported that patients with Graves' disease have a better prognosis; however, reports from many surgeons could not reproduce the same results [10-14]. Patients with Hashimoto's disease and PMC had a recurrence-free survival at 35 years that was better than those preoperatively diagnosed with a benign nodule and PMC [15]. Patients who were preoperatively diagnosed as having any kind of diffuse goiter coexisting with their PMC had recurrence-free survival at 35 years that was better than those preoperatively diagnosed as having a benign nodule and PMC (Fig. 4). When the size of the PMC is compared among the above groups, the PMC of the Graves' disease group and the Hashimoto's thyroiditis group had a maximum dimension of $3.9 \pm 2.3 \mathrm{~mm}$. The PMC of patients with any type of diffuse goiter had a maximum dimension of $4.9 \pm 2.8 \mathrm{~mm}$, and the mean size of the primary PMC tumor of those preoperatively diagnosed with a combination of benign nodule and PMC was $7.4 \pm 2.5 \mathrm{~mm}$. Therefore, the average size of the tumor could be one of the factors in a better prognosis.
Nodal metastasis

Patients who had no gross metastases and those whose metastases were not examined had almost the same recurrence-free survival rate (Fig. 7). Cases in which gross lymph node metastases were found had a substantially worse prognosis. This may suggest that if an experienced surgeon believes that lymph node removal is not indicated because the nodes appear normal, then nodal metastases probably is not present. Alternatively, it may be that central neck nodal metastasis is relatively rare in patients with tumors of this size, and so any deleterious effect is diminished by the large number of node-negative patients included in this group.

\section{Recurrence and death}

Tumor recurred in 73 patients $(3.5 \%)$ over a 35 -year time span. Among them, 68 patients (90.7\%) experienced recurrences in the thyroid bed and/or cervical lymph nodes. A few patients had up to four recurrences. Patients who had recurrence in the neck area could be treated with secondary surgery.

Death resulting from papillary thyroid carcinoma is uncommon and is particularly rare in low-risk patients like PMC [9]. Of 2070 patients who were followed up for $16.5 \pm 7.3$ years, only 12 patients $(0.6 \%)$ died of thyroid cancer.

Invasion

Invasion of the primary tumor beyond the thyroid capsule is one of the prognostic risk factors of papillary cancer of all sizes [6]. When the primary tumor adheres or invades the recurrent nerve or the muscular layer of the esophagus, the recurrence-free survival rate decreases significantly (Figs. 5 and 6).

\section{Surgical treatment}

For treatment of PMC we perform thyroid lobectomy alone when there is no indication beyond the cancer therapy for a bilateral procedure. When an adenoma is present in the opposite lobe of the thyroid, we excise some benign thyroid tissue. We do not perform total thyroidectomy or bilateral thyroid lobectomy when PMC is limited to one lobe. When lymph node metastasis is present in the lateral compartment of the neck, modified radical neck dissection and central compartment neck dissection is mandatory. 
Postoperative adjuvant therapy

In our previous report [6] we recommended TSH suppression to all postoperative PMC patients, but the majority of patients who did not have postoperative hypothyroidism discontinued medication within a few years. We evaluated whether patients who discontinued TSH suppression had a higher incidence of recurrence. We found that there was no statistically significant difference. Because most patients with one intact thyroid lobe have normal levels of T4, we discontinued the practice of prescribing thyroid hormones to postoperative patients with normal thyroid function. Recently, it has been reported that TSH may not be the dominant growth factor for benign and malignant thyroid tumors [16]. This finding confirms our observation. We do not use radioactive iodine unless there is evidence of distant metastasis. In sum, our only postoperative management is to observe the patient once a year with ultrasonography and, if necessary, fine-needle aspiration cytology as well as checking serum thyroglobulin levels. Pelizzo et al. [17] uses more aggressive treatments, including total thyroidectomy and radioactive iodine treatment. However, their recurrence rate is higher than ours within a shorter follow-up period. There are many experts who treat PMC with the same or slightly less aggressive regimen as used for clinical papillary carcinoma $[18,19]$. Furlan et al. [20] use near total thyroidectomy and optionally consider radioactive ablation. We consider thyroid lobectomy or subtotal thyroidectomy without radioactive iodine ablation of residual thyroid tissue as sufficient therapy once unifocal PMC has been established. Similarly, the Institute Gustave-Roussey group believe that loboisthmectomy is the treatment of choice [21]. Peizzo et al. [17] recommended lobectomy plus TSH suppression in 1990. Rodriguez et al. [22] recommended lobectomy alone. However, the treatment of incidental multifocal PMC is still controversial. When other papillary microcarcinomas are found during surgery, the Institute GustaveRoussey group perform total thyroidectomy. However, incidental PMC is often not proven until postoperative histopathology is performed.

\section{Conclusion}

PMC is diagnosed more frequently because of high-quality ultrasonography and fine-needle aspiration cytology. In 2006, PMC represented more than half of papillary carcinoma cases at our institution. Experienced physicians can accurately biopsy PMC as small as $3 \mathrm{~mm}$ in diameter. However, these results confirm that papillary microcarcinoma of the thyroid has an excellent prognosis. Lobectomy may be sufficient therapy in a vast majority of cases. TSH suppression and radioactive iodine ablation of the remaining thyroid tissue is not necessary. Rather, a higher priority should be placed on follow-up with neck ultrasonography because $99.5 \%$ of recurrences first appear in the neck. To resolve clinical questions, fine-needle aspiration cytology can be repeated at regular intervals. Serum thyroglobulin levels can also be informative. The most important lesson of our report is that recurrence can appear even 30 years after primary surgery.

Open Access This article is distributed under the terms of the Creative Commons Attribution Noncommercial License which permits any noncommercial use, distribution, and reproduction in any medium, provided the original author(s) and source are credited.

\section{References}

1. Ito $\mathrm{Y}$, Uruno $\mathrm{T}, \mathrm{Nakano} \mathrm{K}$ et al (2003) An observation trial without surgical treatment in patients with papillary microcarcinoma of the thyroid. Thyroid 13:381-387

2. Kucuk NO, Tari P, Tokmak E et al (2007) Treatment for microcarcinoma of the thyroid-clinical experience. Clin Nucl Med 32:279-281

3. Pelizzo MR, Boschin IM, Toniato A et al (2006) Papillary thyroid microcarcinoma (PTMC): prognostic factors, management and outcome in 403 patients. Eur J Surg Oncol 32:1144-1148

4. Fukunaga FH, Yatani R (1975) Geographic pathology of occult thyroid carcinomas. Cancer 36:1095-1099

5. Grant S, Luttrell B, Reeve T et al (1984) Thyroglobulin may be undetectable in the serum of patients with metastatic disease secondary to differentiated thyroid carcinoma. Follow-up of differentiated thyroid carcinoma. Cancer 54:1625-1628

6. Noguchi S, Yamashita H, Murakami N et al (1996) Small carcinomas of the thyroid. A long-term follow-up of 867 patients. Arch Surg 131:187-191

7. Cady B, Rossi R, Silverman M et al (1985) Further evidence of the validity of risk group definition in differentiated thyroid carcinoma. Surgery 98:1171-1178

8. Chow SM, Law SC, Chan JK et al (2003) Papillary microcarcinoma of the thyroid: Prognostic significance of lymph node metastasis and multifocality. Cancer 98:31-40

9. Vini L, Hyer S, Pratt B et al (1999) Good prognosis in thyroid cancer found incidentally at surgery for thyrotoxicosis. Postgrad Med J 75:169-170

10. Belfiore A, Garofalo MR, Giuffrida D et al (1990) Increased aggressiveness of thyroid cancer in patients with Graves' disease. J Clin Endocrinol Metab 70:830-835

11. Hales IB, McElduff A, Crummer P et al (1992) Does Graves' disease or thyrotoxicosis affect the prognosis of thyroid cancer? J Clin Endocrinol Metab 75:886-889

12. Kasuga Y, Sugenoya A, Kobayashi S et al (1993) The outcome of patients with thyroid carcinoma and Graves' disease. Surg Today 23:9-12

13. Rassael H, Thompson LD, Heffess CS (1998) A rationale for conservative management of microscopic papillary carcinoma of the thyroid gland: a clinicopathologic correlation of 90 cases. Eur Arch Otorhinolaryngol 255:462-467

14. Salvadori B, Del Bo R, Pilotti S et al (1993) "Occult" papillary carcinoma of the thyroid: a questionable entity. Eur J Cancer 29A: $1817-1820$

15. Singh B, Shaha AR, Trivedi H et al (1999) Coexistent Hashimoto's thyroiditis with papillary thyroid carcinoma: impact 
on presentation, management, and outcome. Surgery 126:10701076; discussion 1076-1077

16. Derwahl M, Broecker M, Kraiem Z (1999) Clinical review 101: Thyrotropin may not be the dominant growth factor in benign and malignant thyroid tumors. J Clin Endocrinol Metab 84:829-834

17. Pelizzo MR, Boschin IM, Toniato A et al (2004) Natural history, diagnosis, treatment and outcome of papillary thyroid microcarcinoma (PTMC): a mono-institutional 12-year experience. Nucl Med Commun 25:547-552

18. Sakorafas GH, Stafyla V, Kolettis T et al (2007) Microscopic papillary thyroid cancer as an incidental finding in patients treated surgically for presumably benign thyroid disease. J Postgrad Med 53:23-26
19. Sanders LE, Rossi RL (1995) Occult well differentiated thyroid carcinoma presenting as cervical node disease. World J Surg 19:642-646; discussion 646-647

20. Furlan JC, Bedard Y, Rosen IB (2001) Biologic basis for the treatment of microscopic, occult well-differentiated thyroid cancer. Surgery 130:1050-1054

21. Baudin E, Travagli JP, Ropers J et al (1998) Microcarcinoma of the thyroid gland: the Gustave-Roussy Institute experience. Cancer 83:553-559

22. Rodriguez JM, Moreno A, Parrila P et al (1997) Papillary thyroid microcarcinoma: clinical study and prognosis. Eur J Surg $163: 255-259$ 